\title{
Intracranial epidural hematoma after use of a 3-pin head clamp in a pediatric case with posterior fossa tumor and hydrocephalus: A case report
}

\author{
Mehmet Hakan Şahin ${ }^{1 *}$, Mete Zeynal ${ }^{1}$, Nuh Çağrı Karaavc1 ${ }^{1}$, Mehmet Kürşat Karadağ ${ }^{1}$ \\ ${ }^{1}$ UNIVERSITY OF ATATURK SCHOOL OF MEDICINE, DEPARTMENT OF NEUROSURGERY, ERZURUM, TURKEY
}

\begin{abstract}
The head clamp system is one of the indispensable equipment of neurosurgery in terms of stabilizing the head and positioning it. In addition, in neurosurgery clinics, the use of pin head clamp is required to use the navigation system effectively. In pediatric cases, complications after the use of pins head clamp are rare, as reported in the literature. This paper presents a pediatric patient with posterior fossa tumor and hydrocephalus, who developed depression fracture and epidural hematoma after the use of Mayfield 3-pin clamp head. For this reason, the patient underwent emergency surgery a second time and the epidural hematoma was evacuated to obtain decompression. Epidural bleeding, which is a complication of the pinned clamped head, is frequently described in the literature, often in the pediatric cases accompanied by posterior fossa tumor and hydrocephalus. In this type of pediatric cases, a safe study for the use of pin clamp head is not yet available in the literature.
\end{abstract}

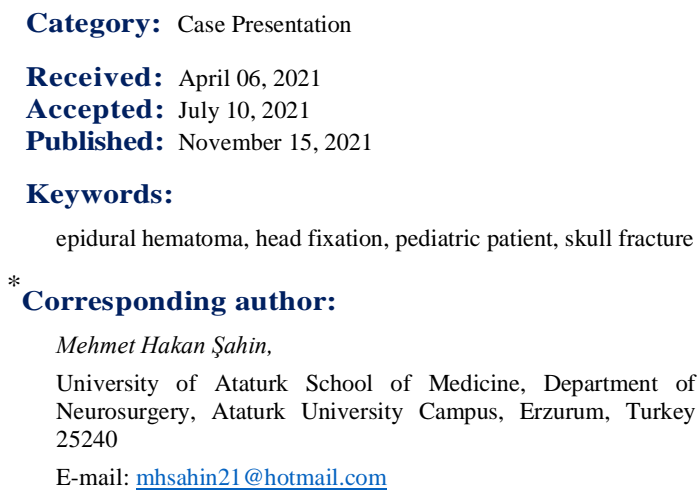

\section{Introduction}

The head clamp system is an important and frequently used application in neurosurgery. Although there are many anatomical trick points during the application, some complications can be seen during or after insertion. The most important of these is intracranial hemorrhage [1,2]. We report a case that developed an epidural hematoma after the use of a head clamp system, which is rarely reported in the literature.

\section{Case Presentation}

A 5-year-old male patient applied to our outpatient clinic with headache for 6 months and unbalanced walking for the last 15 days. His examination included ataxic gait and nystagmus. There was no additional disease and no regular drug use history. The patient was hospitalized and examined.

In the 4th ventricle, a tumor with a slight hypointense in T1-weighted sections of approximately $6 \times 6 \times 6 \mathrm{~cm}$ and a heterogeneous contrast involvement after hyperintense contrast agent injection in T2-weighted sections was observed (Figure 1A). However, T2-weighted, and FLAIR- weighted imaging showed significant dilatation in the 3rd ventricle and lateral ventricles, and views compatible with subependymal CSF migration were observed in the periventricular areas (Figure 1B). Surgical preparations were made by diagnosing posterior fossa tumor and hydrocephalus.

The patient was taken to the operating table in a prone position. After adjusting the Mayfield clamp head pins to pay attention to the anatomical landmarks, the torque screw thread of the Mayfield was compressed by 4 units and stabilized with a pressure of 40 pounds.

External ventricular drainage system was applied from the right coher point. By making this intervention, it was aimed to take precautions both to prevent tonsillar herniation during surgery and for a condition that would cause acute hydrocephalus, such as postoperative bleeding or residual tumor. The tumor was totally resected. During the operation and at the end, there were no findings to suspect an increase in intracranial pressure. The postoperative patient was intubated to intensive care unit and in the third hour of postoperative intensive care, Glaskow Coma Scale: 10, anisocoria and weakness on the left side were observed. The 
patient underwent brain computed tomography (CT) imaging. The right frontotemporal junction had a depression fracture and a right frontal, temporal and parietal epidural hematoma with the widest part of $3 \mathrm{~cm}$ (Figure 1C-1D). The patient was operated urgently, the hematoma was evacuated, and control tomography imaging was performed. The epidural hematoma was evacuated (Figure 1E).

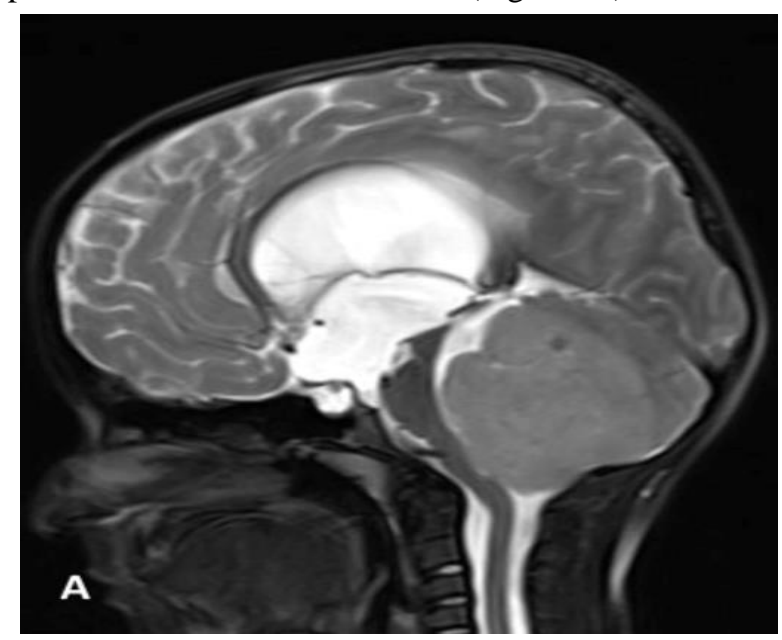

Figure 1A. T2-weighted sagittal brain MR sections show a giant tumor located in the posterior fossa.

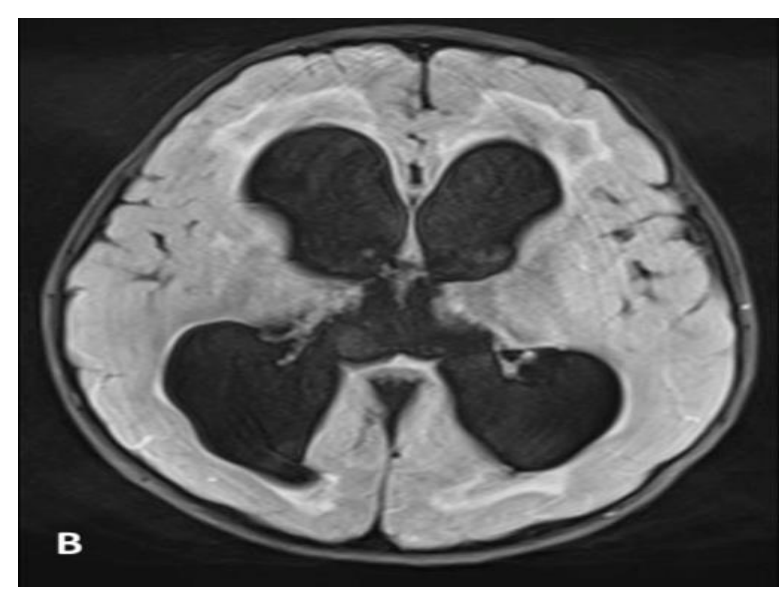

Figure 1B. T2-weighted axial brain MR sections show hydrocephalus and periventricular escape.

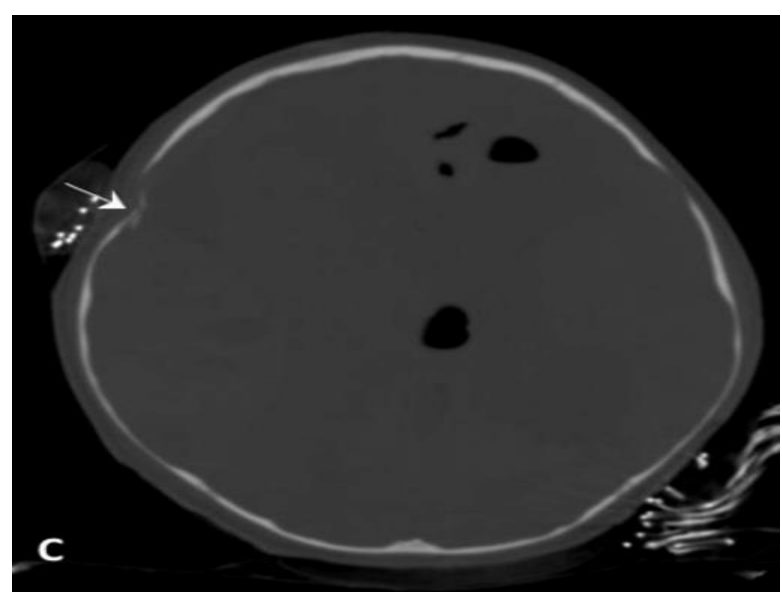

Figure 1C. Postoperative computed tomography shows a depression fracture (white arrow) at the right frontoparietal junction in the bone section.

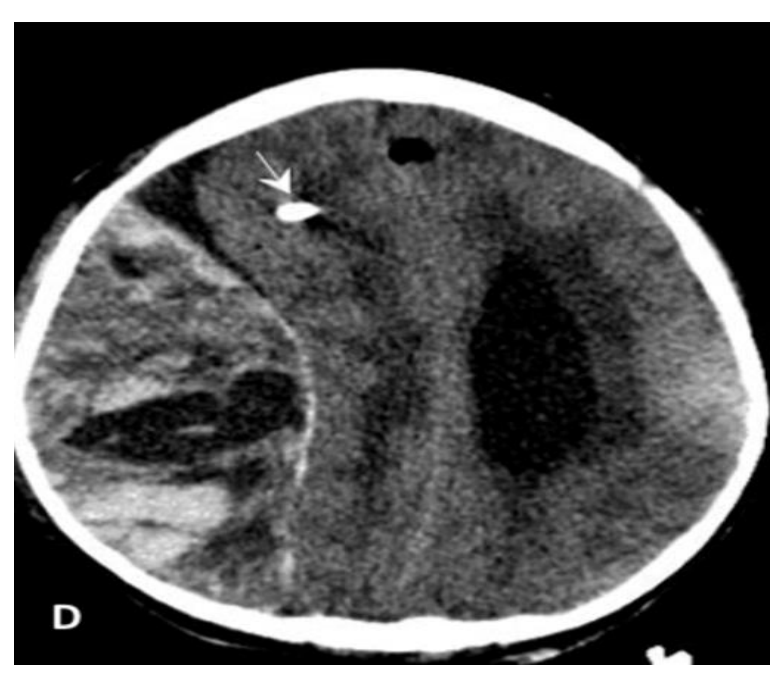

Figure 1D. Postoperative computed tomography shows an epidural hematoma at the right frontoparietal junction in the brain section (the ventricular catheter is shown with the white arrow).

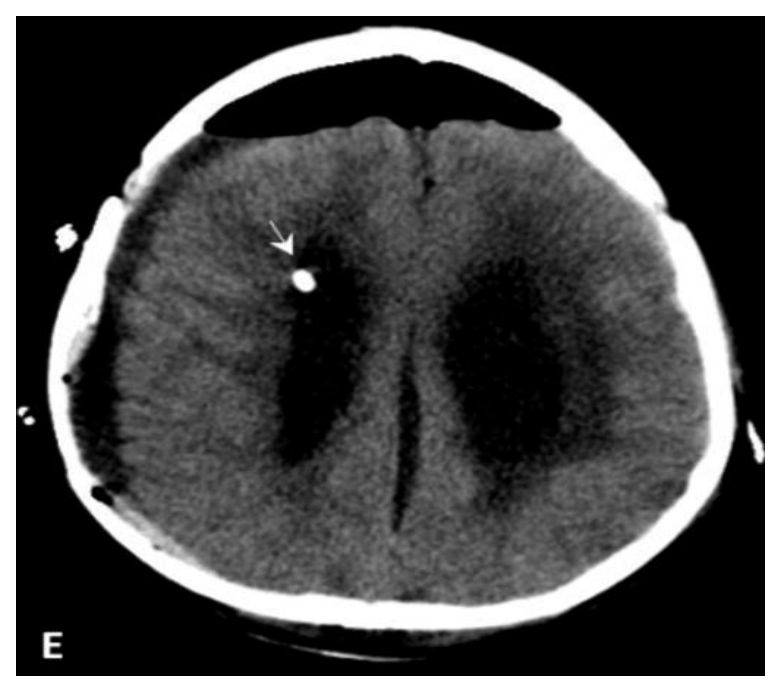

Figure 1E. Postoperative brain tomography of the patient, who was operated after epidural hematoma and depression fracture, has been seen (the ventricular catheter is shown with the white arrow).

\section{Discussion}

The head clamp system is one of the indispensable equipment of neurosurgery in terms of stabilizing the head and positioning it. In addition, the use of pin head clamp in neurosurgery is required to use the navigation system effectively. Its use is very common in our own clinic. Commonly, a 3-pin Mayfield $®$ system or a 4-pin Sugita ${ }^{\circledR}$ system can be used. The pins penetrate the outer cortex layer of the bone and provide stabilization of the head [3,4].

For many years, there are several known principles for the application of the head clamp system application to the patient and surgeon. In 3-pin systems, it is based on the principle of compression with the appropriate pressure, following the selection of a suitable anatomical area between the 2-pin arm and the one-pin counterpart. 
During this procedure, it should be paid attention not to overlap the neurovascular structures and temporal muscle in the skin, which should be anatomically considered in terms of the locations of the head clamp. In addition, in patients with thin skull bone, these areas should be avoided in pin penetration due to its thin structure in dural sinus areas and temporal squamous bone [5-7].

Head clamp use is not recommended for pediatric patients under 3 years old due to the risk of skull fracture. The Mayfield $\AA$ compression pressure we use should be limited to 60-80 pounds for adult patients and 30-40 pounds for pediatric patients over the age of three. In the Sugita fixing system, there is a place for 6 pins and fixation is done with 4 pins. In pediatric cases, it is predicted that 6 pin inputs can be used, and it can be used more safely by aiming to decrease the pressure per pin $[3,7]$.

In a survey study by Berry et al. involving 164 pediatric neurosurgeons, $50 \%$ of surgeons used head clamp for patients under 3 years old, $89 \%$ for patients 3-4 years old, and all of them were 5 years old and older. Again, in this survey, according to the age of the patients, the clamped head was used with a compression pressure of 10-40 pounds. However, it is seen that the surgeons who participated in this study benefited from experience rather than any scientific data regarding this pressure selection [6].

In a study conducted by Aoki et al., 4 pediatric cases ranging from 9 months to 3 years of age were presented. With this method, the plugs belonging to the used antibiotic bottles are inserted into the clamp cap pins. In this way, it has been stated that both the size of the pin is shortened, and the penetrating part is reduced, and the rubber surface area is expanded and possible pin-pon ball fracture is prevented [5].

A number of complications such as pin site infection, depression fracture, air embolism and intracranial epidural hematoma can be seen rarely, especially in pediatric cases, during the attachment of the head clamp [8-10].

Complications of the head clamp system reported as in our case up to now, total 13 pediatric cases have been reported. However, in another scientific study by Berry and col., 54\% of the participants reported 142 complications and half of these complications were epidural hematoma. According to the results of this study, especially in pediatric cases, the complications of the 3 pin head clamps are not as low as expected according to the literature. We think that this difference may occur as a result of surgeons avoiding reporting complications [11-13].

There are several factors able to disrupt the mineral structure of the bone, such as antiepileptic use for more than 2 years, vitamin D deficiency and chronic renal failure in developing complications in head clamp applications [14-16].
In addition, as in our case and in most cases reported to the literature, the association of posterior fossa tumor with chronic intracranial pressure and chronic hydrocephalus increases the risk of fractures. In addition, tumor resection and CSF draining during surgery decreases the intracranial pressure and is expected to increase in a possible bleeding. However, it is believed that, since the posterior fossa tumors are in the prone position, they may bleed secondary to the increase in cerebral venous pressure $[3,17]$.

The general recommendation to prevent complications of 3-pin head clamps in pediatric cases is that it should not be applied under the age of 3 or to use clamp systems containing horseshoe and gel headrest. Preoperative brain tomography can be used to examine the thickness of the lateral edges of the head bone, especially where the pins are often placed. Several studies involving halo fixation show that pins under 2-3 years old can be safely used 2-3 $\mathrm{mm}, 4-6$ years old $4 \mathrm{~mm}$ and 6-13 years old 5-6 mm pins $[6,18]$.

In another aspect, in patients undergoing a 3-pin head clamp, intensive nociceptive stimulation may result in hypertension and tachycardia. This can also increase intracranial pressure and bleeding that may develop. In some studies, remifentanil was found to reduce such stress response by $40 \%$ compared to propofol treatment and reduce the risk. As a result, some anesthetic agents may support safer use of the pin-head clamp [19,20].

In addition, attention should be paid to the anatomical trick points during use of the head clamp and whether there will be a bone crack sound during insertion. Especially in pediatric patients with posterior fossa tumor and hydrocephalus, it should be kept in mind that if herniation occurs despite the fact that the patient is given a good position during the operation and sufficient CSF is evacuated, epidural or subdural hematoma can cause this condition.

In such a situation that may occur, especially in pediatric cases, intraoperative or postoperative early tomography is very important for the diagnosis or exclusion of possible complications [21-23].

\section{Conclusions}

There are methods of use and pressure adjustments based on the experience of pediatric surgeons on how to use pin head clamps in the pediatric population. However, there is no consensus. Biophysical studies are needed to evaluate pediatric case use more objectively. In the literature, many of the pediatric patients with epidural hematoma developing after pin head clamp appear to be patients with posterior fossa located tumor and chronic hydrocephalus. In these cases, with at least known and routine techniques, it is certain that pin head clamps should not be used or should be used very carefully. 


\section{Conflict of interest disclosure}

There are no known conflicts of interest in the publication of this article. The manuscript was read and approved by all authors.

\section{Compliance with ethical standards}

Any aspect of the work covered in this manuscript has been conducted with the ethical approval of all relevant bodies and that such approvals are acknowledged within the manuscript.

\section{References}

1. Ortiz-Ordonez A, Jiménez-Deleg G Sr, Salman S. Fatal Distant Venous Epidural Hematoma Following Posterior Cranial Fossa Surgery in a Twelve-Year old Child. Cureus. 2021 Jan 13;13(1):e12686. doi: 10.7759/cureus. 12686

2. Yu J, Yang H, Cui D, Li Y. Retrospective analysis of 14 cases of remote epidural hematoma as a postoperative complication after intracranial tumor resection. World J Surg Oncol. 2016 Jan 6;14(1):1. doi: 10.1186/s12957-015-0754-8

3. Lee M, Rezai AR, Chou J. Depressed skull fractures in children secondary to skull clamp fixation devices. Pediatr Neurosurg. 1994;21(3):174-7; discussion 178. doi: 10.1159/000120830

4. Vitali AM, Steinbok P. Depressed skull fracture and epidural hematoma from head fixation with pins for craniotomy in children. Childs Nerv Syst. 2008 Aug;24(8):917-23. doi: 10.1007/s00381-008-0621-9

5. Aoki N, Sakai T. Modified application of three-point skull clamp for infants. Neurosurgery. 1989 Oct;25(4):660-2. doi: 10.1097/00006123-19891000000028

6. Berry C, Sandberg DI, Hoh DJ, Krieger MD, McComb JG. Use of cranial fixation pins in pediatric neurosurgery. Neurosurgery. 2008 Apr;62(4):913-8. doi: 10.1227/01.neu.0000318177.95288.cb

7. Domenech-Fernandez P, Yamane J, Domenech J, Barrios C, Soldado-Carrera F, Knorr J, Canavese F. Analysis of skull bone thickness during growth: an anatomical guide for safe pin placement in halo fixation. Eur Spine J. 2021 Feb;30(2):410-415. doi: 10.1007/s00586-020-06367-x

8. Yan HJ. Epidural hematoma following use of a threepoint skull clamp. J Clin Neurosci. 2007 Jul;14(7):6913. doi: 10.1016/j.jocn.2006.02.012

9. Popescu B, Oașă ID, Bertesteanu SV, Balalau C, Manole F, Domuta M, Oancea AL. Strategies to improve activity and results of the head and neck tumor board. J Clin Invest Surg. 2020;5(1):9-12. doi: 10.25083/2559.5555/5.1/9.12
10. Ozen V, Orhan ME. Review of the effects of anesthetic agents used as premedication for patients undergoing electroconvulsive therapy with diagnoses of bipolar disorder or major depression on convulsion, recovery period, and hemodynamic parameters. J Mind Med Sci. 2019;6(2):271-277. doi: 10.22543/7674.62.P271277

11. Berry C, Ley EJ, Tillou A, Cryer G, Margulies DR, Salim A. The effect of gender on patients with moderate to severe head injuries. J Trauma. 2009 Nov; 67(5):950-3. doi: 10.1097/TA.0b013e3181ba3354

12. Xiaoyu W, Guoping L. Surgical treatment of supra- and infratentorial epidural hematoma. Turk Neurosurg. 2013; 23(3):299-303. doi: 10.5137/1019-5149.JTN.5043-11.1

13. Parenrengi MA, Adhiatmadja F, Arifianto MR, Apriawan T, Fauzi AA, Servadei F. Bilateral skull fracture with massive epidural hematoma secondary to pin-type head fixation in a pediatric patient: Case report and review of the literature. Int J Surg Case Rep. 2019;62:43-49. doi: 10.1016/j.ijscr.2019.07.079

14. Matouk CC, Ellis MJ, Kalia SK, Ginsberg HJ. Skull fracture secondary to application of a Mayfield skull clamp in an adult patient: case report and review of the literature. Clin Neurol Neurosurg. 2012 Jul;114(6): 776-8. doi: 10.1016/j.clineuro.2011.12.036

15. Jung JH, Chai YH, Jung S, Kim IY, Jang WY, Moon KS, Kim SK, Chong S, Kim SK, Jung TY. Visual outcome after endoscopic third ventriculostomy for hydrocephalus. Childs Nerv Syst. 2018 Feb;34(2):247255. doi: 10.1007/s00381-017-3626-4

16. Tudorachi NB, Eva I, Dascalu CG, AL-Hiary R, Barbieru B, Paunica M, Motofei C, Moraru AC. The influence of serum calcium and magnesium levels in the radiological evolution of knee osteoarthritis. J Mind Med Sci. 2020;7(2):217-226. doi: 10.22543/7674.72.P217226

17. Jang EA, Lee S, Choi JI, Cho SY. Changes in the hemodynamic parameters between the prone and supine positions measured by an arterial pulse contour cardiac output monitoring system. Anesth Pain Med. 2015;10(4):291-294. doi: 10.17085/apm.2015.10.4.291

18. Kageji T, Nagahiro S, Mizobuchi Y, Nakajima K. Postoperative Hematoma Requiring Recraniotomy in 1149 Consecutive Patients With Intracranial Tumors. Oper Neurosurg (Hagerstown). 2017 Jun 1;13(3):392397. doi: 10.1093/ons/opw045

19. Berger M, Philips-Bute B, Guercio J, Hopkins TJ, James ML, Borel CO, Warner DS, McDonagh DL. A novel application for bolus remifentanil: blunting the hemodynamic response to Mayfield skull clamp placement. Curr Med Res Opin. 2014 Feb;30(2):24350. doi: 10.1185/03007995.2013.855190

20. Liu YH, Hu XB, Yang XM, Wang YW, Deng M. Comparing remifentanil and sufentanil in stress reduction during neurosurgery: a randomised 
controlled trial. Int J Clin Pharm. 2020 Oct;42(5): 1326-1334. doi: 10.1007/s11096-020-01094-1

21. Krishnan P, Kumar SK, Kartikueyan R, Patel SM. Pinsite epidural hematoma: A catastrophic complication of cranial fixation in a child. J Neurosci Rural Pract. 2016 Apr-Jun;7(2):286-9. doi: 10.4103/0976-3147.178661

22. Başar I, Aydın Öztürk P, Tuncer MC, Turan Y, Yilmaz T. Surgical management of sporadic hemangioblastomas located in the posterior fossa of brain. Arch Ital Biol. 2021 Jun 30;159(2):51-63. doi: 10.12871/00039829202121

23. Aleksonis HA, Wier R, Pearson MM, Cannistraci CJ, Anderson AW, Kuttesch JF, Compas BE, Hoskinson KR. Associations among diffusion tensor imaging and neurocognitive function in survivors of pediatric brain tumor: A pilot study. Appl Neuropsychol Child. 2021 Apr; 10(2):111-122. doi: 10.1080/21622965.2019.1613993 